\title{
A FUNCTIONAL MECHANISTIC STUDY OF THE EFFECT OF EMOLLIENTS ON THE STRUCTURE AND FUNCTION OF THE SKIN BARRIER
}

\author{
S.G. Danby ${ }^{1}$, J. Chalmers ${ }^{2}$, K. Brown ${ }^{1}$, H.C. Williams ${ }^{2}$, and M.J. Cork ${ }^{1,3,4}$ \\ ${ }^{1}$ The Academic Unit of Dermatology Research, Department of Infection, Immunity \& \\ Cardiovascular Disease, The University of Sheffield Medical School, Sheffield, UK; \\ ${ }^{2}$ The Centre of Evidence Based Dermatology, University of Nottingham, Nottingham, \\ UK; ${ }^{3}$ The Paediatric Dermatology Clinic, Sheffield Children's Hospital, Sheffield, UK; \\ ${ }^{4}$ Department of Dermatology, Royal Hallamshire Hospital, Sheffield, UK
}

Running Head: Effect of emollients on the skin

Manuscript: 3,925 words, 3 Figures and 1 Table

Corresponding Author: Dr Simon G. Danby, The Academic Unit of Dermatology Research, Department of Infection, Immunity \& Cardiovascular Disease, Faculty of Medicine, Dentistry and Health, The University of Sheffield Medical School, Beech Hill Road, Sheffield S10 2RX, UK. Tel: 01142713843, Fax: 01142712933, Email: s.danby@sheffield.ac.uk

Funding: This study was supported by research funding from Nottingham University Hospitals Charity, who had no involvement in the conduct of the study

Conflict of interest: MJC and SGD have received fees for giving lectures and/or attending advisory boards and unrelated research funding from Almirall, Astellas Pharma, MSD, Johnson \& Johnson and Stiefel-GSK who manufacture emollients. HCW, JC, and KB report no conflicts. The choice of emollients tested in this study was made independent of any commercial funder. 


\section{Key words}

Eczema, skin barrier, emollient, transepidermal water loss, hydration, skin-surface-pH

\section{What's already known about this topic?}

- A skin barrier defect is a primary event in the development of atopic dermatitis (AD)

- Topical therapy to correct this skin barrier defect may prevent AD relapses

- Not all emollients exert a positive effect on the skin barrier. The use of Aqueous cream BP for example damages the skin barrier

\section{What does this study add?}

- We provide evidence that, in contrast to Aqueous cream BP, two commonly prescribed emollients exert no negative effects on the skin barrier, and are therefore suitable for further clinical testing in AD prevention trials

- We also highlight that while these ancillary treatments display clinically important 'emollient' properties they do little to actively improve skin barrier function 


\begin{abstract}
Background

Preventing relapses of atopic dermatitis (AD) through the regular use of topical products to repair the skin barrier defect is an emerging concept. It is still unclear if some commonly used emollients exert a positive effect on the skin barrier.
\end{abstract}

\title{
Objectives
}

To determine the skin barrier effects of emollients commonly prescribed in the UK.

\section{Methods}

Two cohorts of volunteers with quiescent AD undertook observer-blind forearmcontrolled studies. The first (18 volunteers) treated the volar side of one forearm with 2 fingertip units of Doublebase ${ }^{\mathrm{TM}}$ gel twice daily for 4 weeks. The second cohort (19 volunteers) undertook the same regimen using Diprobase ${ }^{\circledR}$ cream. Transepidermal water loss (TEWL), stratum corneum integrity and hydration, skin-surface-pH and redness were determined at the test sites before and after treatment.

\section{Results}

Neither Diprobase ${ }^{\circledR}$ cream nor Doublebase ${ }^{\mathrm{TM}}$ gel significantly affected the underlying skin barrier function. Both emollients were associated with significantly increased skinsurface-pH immediately after application (by $0.8 \pm 0.19$ and $1.0 \pm 0.18$ units respectively), and no erythema. Diprobase ${ }^{\circledR}$ cream artificially and transiently (6 hours) improved permeability barrier function by $2.9-3.1 \mathrm{~g} / \mathrm{m}^{2} / \mathrm{h}$ TEWL and increased skin hydration by 6.0-6.2 units. Doublebase ${ }^{\mathrm{TM}}$ gel, containing humectants, was associated with a greater (between 10.1 and 13.0 units during the first 6 hours) and more sustained increase in hydration, lasting more than 12 hours following repeated use.

\section{Conclusion}

Diprobase ${ }^{\circledR}$ cream and Doublebase ${ }^{\mathrm{TM}}$ gel are not associated with skin barrier harm and appear to be appropriate for AD treatment. Whilst displaying emollient properties, neither formulation displayed an ability to actively improve sustained skin barrier function.

[250/250 words] 


\section{INTRODUCTION}

Atopic dermatitis $(\mathrm{AD})$ is a very common chronic inflammatory skin condition affecting up to $30 \%$ of children and up to $10 \%$ of adults worldwide and appears to be on the increase. ${ }^{1-4}$ The early appearance of AD often heralds the development of other allergic diseases such as food allergy, asthma and allergic rhinitis. ${ }^{5}$ Increasing evidence suggests that a skin barrier defect is the primary event in the development of $\mathrm{AD}$ (reviewed in Danby et al 2010). ${ }^{6,7}$ This raises the possibility that correcting the skin barrier defect may prevent or delay the relapse or development of $\mathrm{AD}{ }^{8}$

Emollient therapy, to ameliorate established $\mathrm{AD}$, is the first line treatment for $\mathrm{AD} .{ }^{9}$ Regular, liberal, use of routinely prescribed emollients significantly reduces the severity of established $\mathrm{AD} .{ }^{10}$ It has been suggested therefore that the routine use of emollients could reduce the risk of $\mathrm{AD}$ relapses, or its initial development, by ameliorating the skin barrier defect. In support of this a randomized controlled trial (RCT) in adult patients with established $\mathrm{AD}$ determined that the regular use of a barrier strengthening 'emollient' could prolong the period of remission compared to no treatment. ${ }^{11}$ Furthermore the results of several pilot studies conducted in neonates at high risk of developing AD have indicated that the daily use of emollients from birth could significantly reduce the risk of developing AD. ${ }^{8,12,13}$ Studies like these have renewed interest in emollients as potential skin barrier enhancing agents. By definition however emollients simply soothe, smooth and hydrate skin. To achieve AD prevention, topical therapy should not simply provide temporary relief, but ideally impart easily sustainable repair of the skin barrier defect in a way that would be acceptable to patients and their carers. ${ }^{8}$

We, and others, have demonstrated that emollients have very different effects on the skin barrier depending on their formulation. ${ }^{14-17}$ In particular aqueous cream BP, containing the anionic surfactant sodium lauryl sulphate (SLS), was found to damage the skin barrier, with related adverse effects reported. ${ }^{18-21}$ On the other hand some emollients, with purported skin barrier enhancing effects, were found to reduce the need for topical corticosteroids, reduce the severity of $\mathrm{AD}$ and delay relapse of the condition. ${ }^{8,11,22}$ In a recent RCT in adult patients an emollient, previously found to strengthen the skin barrier, was shown to increase the period of $\mathrm{AD}$ remission compared to an emollient without 
effects on the skin barrier. ${ }^{23}$ These 'positive' emollients all have complex formulations and contain bioactive ingredients that potentially affect skin barrier homeostasis. ${ }^{24,25}$ It is therefore important to recognize that not all emollients are the same. Just because one emollient reduces the severity of $\mathrm{AD}$ does not mean that all emollients will exhibit such a "class" effect. The heterogeneity of emollient performance has significant implications for the treatment of existing $\mathrm{AD}$, its long-term control, and for prevention strategies in the future. Not surprisingly therefore the Eczema Priority Setting Partnership found that identifying the most effective and safe emollients for treating $\mathrm{AD}$ is a top priority shared by patients and healthcare professionals. ${ }^{26}$ We therefore sought to conduct an independent academic study to determine the effects of commonly used / prescribed emollients in the UK on the skin barrier in order to provide independent evidence for healthcare professionals and to aid the design of future randomized controlled clinical trials focused on eczema control and prevention. In view of the absence of independent evidence on commonly used / prescribed emollients in either adults or children, we chose to conduct this initial mechanistic study in adult patients with quiescent $\mathrm{AD}$, who display a skin barrier defect without visible inflammation. ${ }^{27}$ 


\section{MATERIALS AND METHODS}

\section{Participants}

One cohort of volunteers with healthy skin (cohort 1) and two cohorts of volunteers with quiescent $\mathrm{AD}$ (cohorts 2 and 3) were recruited. Recruitment to all cohorts was open to male and female volunteers, who were recruited on a first come first served basis, assuming they met the specified criteria. Inclusion criteria for cohort 1 included having no current or past signs or symptoms of any chronic skin condition; a Fitzpatrick skintype of I-IV; and being aged between 18 years and over. Inclusion criteria for cohorts 2 and 3 included: a self-reported history of AD, but without any signs or symptoms within the 6 months leading up to participation in the study; a Fitzpatrick skin-type of I-IV; and being aged between 18 and 50 years old. Exclusion criteria for all cohorts included: having a skin condition other than $\mathrm{AD}$, pregnancy, breast-feeding, the use of systemic corticosteroids in the past 12 months, the use of topical anti-inflammatories in the past 6 months, and a known allergy/ hypersensitivity to any of the excipients of the trial preparations. Informed consent was obtained from each participant. All participants received remuneration appropriate for their involvement. The NRES committee East Midlands - Derby formally known as Trent Multicentre Research Ethics Committee (MREC) approved the study, under the project reference 04/MREC/70.

\section{Rationale for emollients selected for testing}

To select the emollients used in this study we were guided by patient feedback collected as part of the Barrier Enhancement for Eczema Prevention (BEEP) eczema prevention feasibility study conducted in the UK and US. ${ }^{8}$ In this study participants were able to choose the emollient intervention from a panel comprising of a topical oil, an ointment, and a cream/gel. In the UK these were: sunflower seed oil, liquid paraffin $50 \%$ in white soft paraffin and Doublebase ${ }^{\mathrm{TM}}$ gel. In the USA these comprised sunflower seed oil, Aquaphor ${ }^{\circledR}$ healing ointment, and Cetaphil ${ }^{\circledR}$ cream. Of the participants, $67.2 \%$ selected

Doublebase $^{\mathrm{TM}}$ gel or Cetaphil ${ }^{\circledR}$ cream indicating a preference for cream/gel formulations, and so we concentrated on this class of emollient. When the parents of infants enrolled onto the trial were offered a selection of emollients creams/gels, they said that they preferred Doublebase ${ }^{\mathrm{TM}}$ gel and Diprobase ${ }^{\circledR}$ cream. In the UK, based upon the 2013 
prescription cost analysis for the NHS, Doublebase ${ }^{\mathrm{TM}}$ gel and Diprobase ${ }^{\circledR}$ cream were the most frequently prescribed emollients (including ointments) jointly accounting for $33 \%$ (16\% and $15 \%$ respectively). ${ }^{28}$ To date no independent evaluation of the effects of these emollients on the structure and function of the skin barrier has been conducted in adults or children. Given their distinct formulations we assessed the effects of these two emollients on the skin barrier in adult patients with quiescent AD.

\section{Single application test}

The forearms (volar face) of the volunteers in cohort 1 were divided into 4 test sites $(4 \times 3$ $\mathrm{cm}$ ) each. Each test site received a single $100 \mu \mathrm{l}$ application of Aqueous cream BP, Diprobase ${ }^{\circledR}$ cream, Doublebase ${ }^{\mathrm{TM}}$ gel (see Table 1 for details) or no treatment so that each treatment was repeated twice per subject (randomized allocation within each forearm using a randomization list generated at www.randomisation.com - product identities were concealed from the investigator and participant using unlabeled packaging). The biophysical properties of the test sites were determined before and at set time points after treatment application. Participates were asked to refrain from washing the test sites until completion of the study.

\section{Treatment regimen for mechanistic studies}

Cohorts 2 and 3 undertook 28-day forearm-controlled observer-blind studies involving self-treatment with Diprobase ${ }^{\circledR}$ cream or Doublebase ${ }^{\mathrm{TM}}$ gel (Table 1). There were two test sites per volunteer, one on each forearm (volar side, $3 \mathrm{~cm}$ below elbow flexure to $3 \mathrm{~cm}$ above the wrist). Each participant was asked to apply 2 finger-tip units of the respective emollient to one forearm and no treatment to the other (randomised allocation) twice daily (at least 6 hours apart) for 28-days. Prior to, and (12-20 hours) following the cessation of, treatment the biophysical properties of the test sites were assessed to determine the effect of the treatment on the underlying condition of the skin barrier. Care was taken to ensure that no emollient residues remained on the skin prior to testing by dry wiping with cotton wool as required.

\section{Biophysical measurements}

Transepidermal Water Loss (TEWL) measurements were performed using an AquaFlux AF200 condensing chamber probe (Biox Systems Ltd., London, UK). Redness, skin 
surface $\mathrm{pH}$ and capacitance were measured using a Mexameter MX18, a Skin-Surface-pH Meter PH905, and a Corneometer CM825 respectively (CK electronic GmbH, Cologne, Germany). All assessments were performed in a room maintained at $21 \pm 2^{\circ} \mathrm{C}$ and $38-50 \%$

relative humidity according to published guidelines. ${ }^{29}$ All test sites were acclimatised to room conditions for 20 minutes before assessment. Tape-stripping, to experimentally disrupt the SC, was performed as previously described. ${ }^{27}$ Determination of the amount of protein removed by tape-stripping was based on the IR absorbance (SquameScan 850A, CuDerm, Dallas, USA) of tape-strips in accordance with published methodology. ${ }^{30}$ Total stratum corneum (SC) thickness $(\mathrm{H})$ was estimated, from the relationship between the cumulative amount of protein removed and TEWL based on Fick's first law. ${ }^{31}$

\section{SC protease activity}

Assessment of protease activity was made on samples comprising three consecutive tapestrips as previously described. ${ }^{30}$ Caseinolytic, chymotrypsin-like and trypsin-like activities were determined using EnzCheck ${ }^{\circledR}$ (Life Technologies Ltd., Paisley, UK), MeOSuc-Arg-Pro-Tyr-AMC (Peptide Protein Research Ltd, Funtley, UK), and Boc-PheSer-Arg-AMC (Bachem, Bubendorf, Switzerland) substrates respectively.

\section{Data analysis}

The results were analysed in Prism v6.01 (Graphpad Software Inc., CA, USA). The significance threshold was $p<0.05$. Results are presented as mean \pm standard error of the mean (SEM). 


\section{RESULTS}

\section{The effect of a single application of three different emollients on the biophysical properties of the skin}

Single applications $(100 \mu \mathrm{l})$ of Aqueous cream, Diprobase ${ }^{\circledR}$ cream, Doublebase ${ }^{\mathrm{TM}}$ gel, and no treatment as a control, were made to separate sites on each forearm of 4 participants with healthy skin (cohort 1, mean age $38 \pm 5$ years, 3 male). Figure 1 illustrates the change in the biophysical properties of the skin over 24 hours following

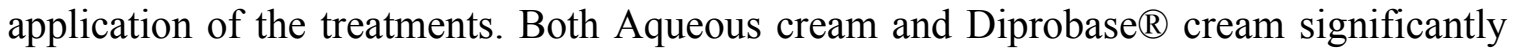
reduced TEWL by approximately $3 \mathrm{~g} / \mathrm{m}^{2} / \mathrm{h}$ for at least 6 hours. After 6 hours the effect diminished (TEWL reduced by 2.070 and $1.968 \mathrm{~g} / \mathrm{m}^{2} / \mathrm{h}$ respectively) but remained significant. Doublebase ${ }^{\mathrm{TM}}$ gel lowered TEWL by a significantly reduced extent, reaching a decrease of $1.850 \mathrm{~g} / \mathrm{m}^{2} / \mathrm{h}$ only after 24 hours. All treatment applications elevated skin hydration, however there were significant differences between the effects of the emollients. Doublebase ${ }^{\mathrm{TM}}$ gel significantly elevated skin hydration by between $10.083 \pm 2.329$ and $13.043 \pm 2.313$ capacitance units during the first 6 hours. By comparison the effects of Diprobase ${ }^{\circledR}$ cream and Aqueous cream were less pronounced, with the increase in hydration reaching $6.196 \pm 1.002$ and $7.836 \pm 1.667$ capacitance units respectively at their peak during the first 6 hours. After 24 hours the hydrating effects of all three emollients were similar. All three treatments significantly elevated skin surface $\mathrm{pH}$ compared to the control, with the highest increase observed for Doublebase ${ }^{\mathrm{TM}}$ gel of $0.976 \pm 0.179$ units (on average) compared to $0.844 \pm 0.186$ units for Diprobase $\mathbb{R}$ cream and $0.823 \pm 0.135$ units for Aqueous cream 10 minutes after application. The $\mathrm{pH}$ of the skin reduced steadily over the following 24 hours, at which time only the sites treated with Diprobase cream were statistically different from the untreated control.

\section{The effect of 28-days treatment with Diprobase ${ }^{\circledR}$ cream on the skin barrier}

Nineteen volunteers with quiescent AD (cohort 2, mean age $33 \pm 2$ years, 14 female) applied an average of $0.96 \pm 0.07 \mathrm{~g}$ Diprobase ${ }^{\circledR}$ cream to one forearm twice per day for 28 days. The other forearm was left untreated as a control. Figure 2 illustrates the biophysical properties of the test sites 12-20 hours following the last application of emollient. No emollient residues were evident on the test sites at the point of testing. At 
this point the transient occlusive effects of the emollients on TEWL have passed and TEWL values reflect the effect of the treatments on the underlying properties of the barrier. No effect of the treatment on TEWL was observed indicating that treatment with Diprobase ${ }^{\circledR}$ cream does not adversely affect skin barrier function. Upon challenge by tape-stripping to assess the integrity of the SC, TEWL was found to be marginally elevated on sites treated with Diprobase ${ }^{\circledR}$ compared to the untreated control sites (Fig 2b), suggesting that the underlying condition of the SC has been adversely affected, albeit to a small degree. There was only a small decrease in SC cohesion (increased protein removed after 3 tape-strips) and no change in estimated SC thickness on the treated compared to the control sites. In agreement with the effects of a single application of Diprobase ${ }^{\circledR}$ cream, hydration was not affected, but skin surface $\mathrm{pH}$ was significantly higher on the treated sites $(4.97 \pm 0.0697$ units) compared to the untreated control sites $(4.79 \pm 0.0561)$. There were no significant differences in skin redness indicating an absence of visible erythema. The effect of treatment with Diprobase ${ }^{\circledR}$ cream on 3 types of SC protease activities associated with skin barrier breakdown and cutaneous inflammation was determined ex vivo, and revealed a significant effect of chymotrypsinlike protease activity, but not trypsin-like or broad-spectrum protease activity.

\section{The effect of 28-days treatment with Doublebase ${ }^{\text {TM }}$ gel on the skin barrier}

Eighteen volunteers with quiescent AD (cohort 3, mean age 29 \pm 8 years, 14 female) applied an average of $1.64 \pm 0.14 \mathrm{~g}$ Doublebase ${ }^{\mathrm{TM}}$ gel to one forearm twice per day for 28 days. The other forearm was left untreated as a control. The amount of Doublebase ${ }^{\mathrm{TM}}$ gel applied was significantly greater than the amount of Diprobase ${ }^{\circledR}$ cream applied despite the same instructions being issued to the volunteers, and indicates that the container type and consistency of the emollient play a role in the amount of product applied even when the same directions are issued. Figure 3 illustrates the biophysical properties of the test sites 12-20 hours following the last application of emollient. No emollient residues were evident on the test sites at the point of testing. Doublebase ${ }^{\mathrm{TM}}$ gel did not significantly affect basal TEWL, indicating that treatment has no negative effects on skin barrier function. Upon tape-stripping of the skin however, TEWL increased at a significantly

slower rate on the sites treated with Doublebase ${ }^{\mathrm{TM}}$ gel compared to the control sites. The amount of protein removed by tape-stripping was also significantly and consistently 
lower on treated sites compared to the control across the depth of the SC, indicating that the skin is more resistant to disruption by tape-stripping. SC thickness was unaffected by the treatment. Skin hydration was significantly increased from $33.5 \pm 1.44$ units before treatment to $46.67 \pm 1.51$ units $12-20$ hours after cessation of treatment with Doublebase ${ }^{\mathrm{TM}}$ gel. There was no significant change in skin hydration on the control site. Skin surface $\mathrm{pH}$ was also significantly increased from 5.00 to 5.234 units on the treated sites, but not on the control sites. There were no significant differences between the Doublebase ${ }^{\mathrm{TM}}$ gel treated sites and the controls for skin redness or any of the ex vivo protease activities tested for. 


\section{DISCUSSION}

\section{Main Findings}

In individuals with healthy skin, a single application of Diprobase ${ }^{\circledR}$ cream, Aqueous cream BP, and to a lesser extent Doublebase ${ }^{\mathrm{TM}}$ gel reduced TEWL demonstrating that they transiently occlude the skin, thereby creating an artificial barrier. The degree of restoration was greatest immediately after application, and declined after 6 hours highlighting the need for regular application. The findings support current guidance to apply emollients 2-4 times daily. ${ }^{9}$ Adherence to this guidance is associated with a greater treatment effect. ${ }^{10}$ All emollients tested also transiently (between 6 and 24 hours) increased stratum corneum hydration, an effect associated with improved permeability barrier function. ${ }^{32}$ Doublebase $^{\mathrm{TM}}$ gel was associated with a 2-fold greater increase in hydration compared to Diprobase ${ }^{\circledR}$ and Aqueous cream, an effect most likely related to the glycerol in Doublebase ${ }^{\mathrm{TM}}$ gel. $^{32}$ Glycerol is a humectant, previously found to hydrate the stratum corneum. All three emollients transiently elevated skin surface $\mathrm{pH}$ by more than 0.5 units for at least 3 hours.

Following treatment twice daily for 28-days in patients with quiescent $\mathrm{AD}$, neither Diprobase ${ }^{\circledR}$ cream nor Doublebase ${ }^{\mathrm{TM}}$ gel significantly affected the underlying barrier function of the skin (indicated by no change in baseline TEWL), assessed 12-24 hours following the last application. This demonstrates that the restoration of permeability barrier function seen for these emollients is both artificial and transient. Both emollients were associated with significantly increased skin surface $\mathrm{pH}$ for a sustained period, but induced no erythema under the conditions tested. The use of Doublebase ${ }^{\mathrm{TM}}$ gel, but not Diprobase ${ }^{\circledR}$ cream, led to a sustained increase in SC hydration, in agreement with the findings after a single application. The effects of Aqueous cream BP have been reported previously. ${ }^{18,20}$

\section{How this study fits in with existing evidence}

The emollient Aqueous cream was recently found to elevate TEWL by an average of 2.44 $\mathrm{g} / \mathrm{m}^{2} / \mathrm{h}(24 \%)$ following a similar treatment regimen in a similar population (quiescent AD). ${ }^{20}$ Following topical treatment with Aqueous cream, the SC was also found to be thinner, which was attributed to an increased rate of desquamation. ${ }^{18}$ In support of this, ex 
vivo activities of proteases engaged in desquamation were found to be elevated following treatment with Aqueous cream, and the surface area and maturity of the uppermost corneocytes was reduced suggesting premature shedding. ${ }^{19}$ These negative effects of Aqueous cream are linked to the presence of SLS as a surfactant/emulsifier in the formulation. SLS is a standard skin irritant used in patch testing, and is known to increase protease-mediated degradation of the skin. $^{33}$ The fact that treatment with neither Diprobase ${ }^{\circledR}$ cream nor Doublebase ${ }^{\mathrm{TM}}$ gel reduced skin barrier function or SC thickness in this study strongly suggests that the SLS in Aqueous cream is responsible for its negative effects, and that the emulsifiers in Diprobase ${ }^{\circledR}$ cream and Doublebase ${ }^{\mathrm{TM}}$ gel are significantly less damaging.

It is notable that while neither Diprobase ${ }^{\circledR}$ cream or Doublebase ${ }^{\mathrm{TM}}$ gel damaged the skin barrier, they also did not improve the underlying condition of the skin barrier. Moreover skin surface $\mathrm{pH}$ was increased significantly following treatment with Diprobase ${ }^{\circledR}$ cream and Doublebase ${ }^{\mathrm{TM}}$ gel, by almost 1 unit immediately following a single application in healthy patients, and by approximately 0.2 units 12-20 hours following the last application of a 28-day regimen in patients with quiescent $A D$. Diprobase ${ }^{\circledR}$ cream and Doublebase $^{\mathrm{TM}}$ gel have a $\mathrm{pH}$ of 4.9 and 7.1 respectively. The elevation of skin surface $\mathrm{pH}$ after application of these emollients reflects an adaption of the skin surface properties to those of the topical product. Despite the difference in $\mathrm{pH}$ between the products the effects on skin surface $\mathrm{pH}$ were similar. The persistence of product components on the skin, such as acids or buffers, likely accounts for the affects observed after 12-20 hours following application, although no surface residue of emollient was evident at this time-point. These changes are similar in scale to the differences between healthy and AD skin, between non-lesional and lesional AD skin, and between the skin of filaggrin loss-offunction mutation associated and non-associated AD skin. ${ }^{34,35}$ The $\mathrm{pH}$ of the SC plays an important central role in skin barrier homeostasis and AD pathophysiology. Elevated SC serine protease activity, including chymotrypsin-like and trypsin-like activities is just one effect of elevated SC $\mathrm{pH}$. What is not clear from the data presented here is the depth of the $\mathrm{SC}$ to which $\mathrm{pH}$ is disrupted and what the clinical consequences of this are. In the case of Diprobase ${ }^{\circledR}$ cream, a marginal reduction in SC integrity was seen upon tape stripping (elevated TEWL in response to tape-stripping), albeit not to the same extent as 
observed for treatment with Aqueous cream. This coincided with the increase in chymotrypsin-like protease activity. It is reasonable to speculate therefore that the reduction in SC integrity results from $\mathrm{pH}$-induced changes in the rate of desquamation/proteolytic degradation of the corneodesmosomal junctions between corneocytes. Protease activity was broadly increased after treatment with Doublebase ${ }^{\mathrm{TM}}$ gel, but this wasn't found to be significant and skin barrier integrity appeared to be improved. A functional consequence of elevated $\mathrm{pH}$ was therefore not observed for Doublebase $^{\mathrm{TM}}$ gel, yet this could have been concealed. The reduction of skin surface $\mathrm{pH}$ and SC serine protease activity, through the use of buffers and some emollients (comprising buffers and acids), has been shown to elicit positive effects on the skin, such as reduced TEWL. ${ }^{36,37}$ This suggests that the inability of the emollients tested here to control SC pH limits theirs beneficial effects on the skin.

\section{The role of humectants in emollients}

Humectants, like glycerol found in Doublebase ${ }^{\mathrm{TM}}$ gel, are added to emollients to improve their capacity to hydrate the skin. In line with this, skin hydration was $30 \%$ higher $12-20$ hours following the last application of a 28-day treatment regimen with Doublebase ${ }^{\mathrm{TM}}$ gel; achieving a level of hydration sufficient to restore xerotic AD skin to healthy levels of hydration. ${ }^{34}$ The degree of hydration was dependent on the length of time since the last application and the number of treatments made, based on the increased level of hydration observed following the 28-day treatment regimen compared to the single application of gel. Care is required when interpreting the hydration data obtained using the capacitance method because surface contaminants can affect the measurements. Whilst challenging to avoid following short treatment times, every care was taken to ensure surface residues were not present 12-20 hour post-treatment time-point following the 28-day treatment regimen. Similar findings have been reported for other emollients formulated with different humectants. ${ }^{38,39}$ The role of the humectant is highlighted by the absence of increased hydration 12-20 hours following a similar treatment regimen with Diprobase ${ }^{\circledR}$ cream, which does not contain a humectant. However it should be noted that increased amounts of Doublebase ${ }^{\mathrm{TM}}$ gel compared to Diprobase ${ }^{\circledR}$ cream tended to be applied by participants who were self-treating. Under controlled conditions, a single application of $100 \mu 1$ Diprobase ${ }^{\circledR}$ cream in healthy individuals was observed to hydrate the skin 
transiently, but to a significantly reduced extent compared to the application of an identical amount of Doublebase ${ }^{\mathrm{TM}}$ gel. Skin occlusion is the mechanism by which nonhumectant emollients hydrate the skin. Evidencing this, a transient (lasting between 6 and 24 hours) reduction in TEWL by approximately $3 \mathrm{~g} / \mathrm{m}^{2} / \mathrm{h}$ following application of

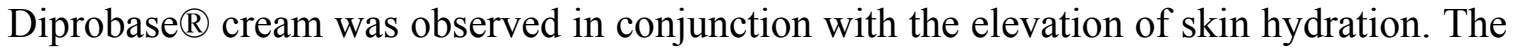
occlusivity of Doublebase $^{\mathrm{TM}}$ gel was not as marked; whether this is due to reduced skin occlusion by Doublebase $^{\mathrm{TM}}$ gel and/or the presence of added glycerol requires further investigation. ${ }^{40}$ Humectants such as glycerol are hygroscopic, and so increase water levels within the SC. Following increases in SC humectant levels TEWL can increase as a result of increased evaporation from the larger store of water.

\section{Conclusion}

The finding that topical treatment with Aqueous cream adversely affects the skin barrier, and in doing so could potentially prolong or exacerbate $\mathrm{AD}$, highlighted the need to determine the safety and appropriateness of other emollients as treatments for AD. We conclude that, unlike Aqueous cream, both Diprobase ${ }^{\circledR}$ cream and Doublebase ${ }^{\mathrm{TM}}$ gel are safe and appropriate for AD treatment as topical leave-on therapy in adults displaying a skin barrier defect. At birth the skin barrier is sub-optimal, and takes a number of months to years to reach adult-like status. ${ }^{41}$ With this in mind the safety of these emollients should also be assessed in neonatal and infant skin. Notably the emollients tested did not display the barrier-strengthening properties reported for some other emollients in the literature. Given that the broad differences observed between infant and adult skin are similar to the differences observed between adults with and without eczema, it is unlikely that a treatment unable to strengthen the barrier in adults could strengthen it in infants and neonates. ${ }^{41}$ This finding highlights that not all emollients are the same, and that effects on the underlying skin barrier represent a key distinguishing factor. A direct comparison of a barrier-strengthening topical product with an emollient without skin barrier effects revealed a significant difference in their ability to control AD. $^{23}$ The humectant glycerol, which differentiates Doublebase ${ }^{\mathrm{TM}}$ gel from Diprobase ${ }^{\circledR}$ cream, appears to significantly enhance the hydrating effects of Doublebase ${ }^{\mathrm{TM}}$ gel. Further clinical testing is required to determine whether Doublebase ${ }^{\mathrm{TM}}$ gel and Diprobase ${ }^{\circledR}$ 
cream differ in their clinical efficacy and to assess the clinical benefit of added humectants per se beyond improved SC hydration. 


\section{TABLES}

Table 1: Emollients used in this study

\begin{tabular}{|c|c|c|c|}
\hline & Aqueous cream BP & Diprobase ${ }^{\circledR}$ cream & Doublebase ${ }^{T M}$ gel \\
\hline Container: & $500 \mathrm{~g}$ tub & $50 \mathrm{~g}$ tube & $500 \mathrm{~g}$ pump dispenser \\
\hline \multirow[t]{2}{*}{ Manufacturer: } & Ecolab, UK & Merck Sharp \& Dohme & Dermal Laboratories, \\
\hline & & Limited, UK & UK \\
\hline \multirow[t]{2}{*}{ Occlusives: } & Liquid paraffin & White soft paraffin & Liquid paraffin \\
\hline & White soft paraffin & Liquid paraffin & \\
\hline Humectants: & & & Glycerol \\
\hline \multirow[t]{3}{*}{ Surfactants/emulsifiers*: } & Cetostearyl alcohol & Cetostearyl alcohol & Isopropyl myristate \\
\hline & Sodium lauryl sulphate & Macrogol cetostearyl & Sorbitan laurate \\
\hline & & ether & Triethanolamine \\
\hline \multirow{6}{*}{$\begin{array}{l}\text { Other ingredients: } \\
\text { (including stabilizers } \\
\text { and preservatives) }\end{array}$} & Phenoxyethanol & Chlorocresol & Carbomer \\
\hline & Purified water & Sodium dihydrogen & Phenoxyethanol \\
\hline & & phosphate & Purified water \\
\hline & & Sodium hydroxide & \\
\hline & & Phosphoric acid & \\
\hline & & Purified water & \\
\hline Product pH & $7.34 \pm 0.009$ & $4.92 \pm 0.003$ & $7.13 \pm 0.002$ \\
\hline
\end{tabular}

*These may also be used as skin softening agents 


\section{FIGURE LEGENDS}

Figure 1: The effect of a single application of Aqueous cream (open circles), Diprobase ${ }^{\circledR}$ cream (closed squares) and Doublebase ${ }^{\mathrm{TM}}$ gel (open diamonds) on skin barrier function (TEWL, panel a), skin hydration (capacitance, panel b), and skin surface $\mathrm{pH}$ (panel c). Results are presented as the change compared to untreated skin. There are 8 repeats of each treatment in 4 subjects. For all three measured parameters a significant effect of the treatment and the interaction between the treatment and time-post washing was found $(p<0.05,2$-way repeated measures ANOVA). Symbols $(\alpha, \beta, \gamma)$ indicate the results of a Dunnett post-test comparing all treatments to the untreated control.

Figure 2: The effect of 28-days treatment with Diprobase ${ }^{\circledR}$ cream, compared to no treatment (NTC), on the biophysical and biological properties of the skin in people with quiescent AD. (a) Skin barrier function, (b) skin hydration, (c) skin surface $\mathrm{pH}$, (d) objective erythema, (e) SC integrity measured as TEWL in conjunction with tapestripping, (f) SC cohesion (protein removed by tape-stripping), (g) estimated SC thickness, (h) ex vivo SC broad-spectrum caseinolytic protease activity, (g) chymotrypsin-like protease activity, (h) and trypsin-like activity. TEWL, capacitance, skin surface $\mathrm{pH}$ and redness were compared with ANCOVA using baseline (Day 0) values as the covariant. SC integrity and cohesion were assessed using a repeated measures 2-way ANOVA. Both the treatment $(p<0.0001)$ and the number of tape-strips significantly ( $p=0.0002)$ affected SC integrity, but not the interaction between the two (n.s.). The number of tape-strips $(p<0.0001)$ and the interaction between tape-strip number and treatment $(p=0.0009)$ affected SC cohesion, but not the treatment independently (n.s.). SC thickness and caseinolytic, chymotrypsin-like and trypsin-like activities were compared using a paired t-test. *Significant differences using either a ttest (a-d and g-j) or Bonferroni post-test (e and f). n.s., not significant.

Figure 3: The effect of 28-days treatment with Doublebase ${ }^{\mathrm{TM}}$ gel, compared to no treatment (NTC), on the biophysical and biological properties of the skin in people with quiescent AD. (a) Skin barrier function, (b) skin hydration, (c) skin surface pH, (d) objective erythema, (e) SC integrity measured as TEWL in conjunction with tapestripping, (f) SC cohesion (protein removed by tape-stripping), (g) estimated SC 
thickness, (h) ex vivo SC broad-spectrum caseinolytic protease activity, (g) chymotrypsin-like protease activity, (h) and trypsin-like activity. TEWL, capacitance, skin surface $\mathrm{pH}$ and redness were compared with ANCOVA using baseline (Day 0) values as the covariant. SC integrity and cohesion were assessed using a repeated measures 2-way ANOVA. The treatment $(p=0.0003)$, the number of tape-strips $(p<0.0001)$ and the interaction between the 2 factors significantly $(p<0.0001)$ affected SC integrity. The treatment $(p<0.0001)$, the number of tape-strips $(p<0.0001)$ and the interaction between the 2 factors significantly $(p<0.0001)$ affected SC cohesion. SC thickness and caseinolytic, chymotrypsin-like and trypsin-like activities were compared using a paired t-test. *Significant differences using either a t-test (a-d and $\mathrm{g}$-j) or Bonferroni post-test (e and f). n.s., not significant. 


\section{ACKNOWLEDGEMENTS}

We are grateful to all our volunteers who have given up their time to take part in our studies. Thanks also go to Les Hunter for the recruitment of volunteers that participated in this study. 


\section{REFERENCES}

1 Odhiambo JA, Williams HC, Clayton TO, et al. Global variations in prevalence of eczema symptoms in children from ISAAC Phase Three. J Allergy Clin Immunol $2009 ; 124: 1251-8$ e23.

2 Williams H, Stewart A, von Mutius E, et al. Is eczema really on the increase worldwide? J Allergy Clin Immunol 2008; 121:947-54 e15.

3 Pesce G, Marcon A, Carosso A, et al. Adult eczema in Italy: prevalence and associations with environmental factors. J Eur Acad Dermatology Venereol $2014 ;: \mathrm{n} / \mathrm{a}-\mathrm{n} / \mathrm{a}$.

4 Silverberg JI, Hanifin JM. Adult eczema prevalence and associations with asthma and other health and demographic factors: A US population-based study. J Allergy Clin Immunol 2013; 132:1132-8.

5 Punekar YS, Sheikh A. Establishing the sequential progression of multiple allergic diagnoses in a UK birth cohort using the General Practice Research Database. Clin Exp Allergy 2009; 39:1889-95.

6 Danby SG, Cork MJ. A New Understanding of Atopic Dermatitis : The Role of Epidermal Barrier Dysfunction and Subclinical Inflammation. J Clin Dermatology $2010 ; 1: 33-46$.

7 Kelleher M, Dunn-Galvin A, Hourihane JO, et al. Skin barrier dysfunction measured by transepidermal water loss at 2 days and 2 months predates and predicts atopic dermatitis at 1 year. J Allergy Clin Immunol 2015; :1-7.

8 Simpson EL, Chalmers JR, Hanifin JM, et al. Emollient enhancement of the skin barrier from birth offers effective atopic dermatitis prevention. J Allergy Clin Immunol 2014; 134:818-23.

9 Lewis-Jones S, Cork MJ, Clark C, et al. Atopic eczema in children - Guideline consultation: A systematic review of the treatments for atopic eczema and guideline for its management. 2007.URL 
http://guidance.nice.org.uk/page.aspx?o=434713.

10 Cork MJ, Britton J, Butler L, et al. Comparison of parent knowledge, therapy utilization and severity of atopic eczema before and after explanation and demonstration of topical therapies by a specialist dermatology nurse. $\mathrm{Br} J$ Dermatol 2003; 149:582-9.

11 Wirén K, Nohlgård C, Nyberg F, et al. Treatment with a barrier-strengthening moisturizing cream delays relapse of atopic dermatitis: a prospective and randomized controlled clinical trial. J Eur Acad Dermatol Venereol 2009; 23:1267-72.

12 Simpson EL, Berry TM, Brown PA, Hanifin JM. A pilot study of emollient therapy for the primary prevention of atopic dermatitis. J Am Acad Dermatol 2010; 63:587-93.

13 Horimukai K, Morita K, Narita M, et al. Application of moisturizer to neonates prevents development of atopic dermatitis. J Allergy Clin Immunol 2014; 134:82430.e6.

14 Held E, Lund H, Agner T. Effect of different moisturizers on SLS-irritated human skin. Contact Dermatitis 2001; 44:229-34.

15 Buraczewska I, Berne B, Lindberg M, et al. Changes in skin barrier function following long-term treatment with moisturizers, a randomized controlled trial. $\mathrm{Br}$ J Dermatol 2007; 156:492-8.

16 Zachariae C, Held E, Johansen JD, et al. Effect of a moisturizer on skin susceptibility to NiCl2. Acta Derm Venereol 2003; 83:93-7.

17 Held E, Sveinsdottir S, Agner T. Effect of long-term use of moisturizer on skin hydration, barrier function and susceptibility to irritants. Acta Derm Venereol 1999; 79:49-51.

18 Tsang M, Guy RH. Effect of Aqueous Cream BP on Human Stratum Corneum In Vivo. Br J Dermatol 2010; 163:954-8. 
19 Mohammed D, Matts PJ, Hadgraft J, Lane ME. Influence of Aqueous Cream BP on corneocyte size, maturity, skin protease activity, protein content and transepidermal water loss. Br J Dermatol 2011; 164:1304-10.

20 Danby SG, Al-Enezi T, Sultan a, et al. The effect of aqueous cream BP on the skin barrier in volunteers with a previous history of atopic dermatitis. $\mathrm{Br} \mathrm{J}$ Dermatol 2011; 165:329-34.

21 Cork BMJ, Timmins J, Holden C, et al. An audit of adverse drug reactions to aqueous cream in children with atopic eczema. Pharm J 2003; 271:746-7.

22 Grimalt R, Mengeaud V, Cambazard F. The steroid-sparing effect of an emollient therapy in infants with atopic dermatitis: a randomized controlled study. Dermatology 2007; 214:61-7.

23 Åkerström U, Reitamo S, Langeland T, et al. Comparison of moisturizing creams for the prevention of atopic dermatitis relapse: A randomized double-blind controlled multicentre clinical trial. Acta Derm Venereol 2015; 95:587-92.

24 Grether-Beck S, Felsner I, Brenden H, et al. Urea uptake enhances barrier function and antimicrobial defense in humans by regulating epidermal gene expression. $J$ Invest Dermatol 2012; 132:1561-72.

25 Buraczewska I, Berne B, Lindberg M, et al. Moisturizers change the mRNA expression of enzymes synthesizing skin barrier lipids. Arch Dermatol Res 2009; 301:587-94.

26 Batchelor JM, Ridd MJ, Clarke T, et al. The eczema Priority Setting Partnership: A collaboration between patients, carers, clinicians and researchers to identify and prioritize important research questions for the treatment of Eczema. Br J Dermatol 2013; 168:577-82.

27 Danby S, Chittock J, Brown K, et al. The Effect Of Tacrolimus Compared To Betamethasone Valerate On The Skin Barrier In Volunteers With Quiescent Atopic Dermatitis. Br J Dermatol 2014; 170:914-21. 
28 Health and Social Care Information Centre. Prescription cost analysis - England 2013. Hscic 2013.URL http://www.hscic.gov.uk/catalogue/PUB13887.

29 Pinnagoda J, Tupker RA, Agner T, Serup J. Guidelines for transepidermal water loss (TEWL) measurement. A report from the Standardization Group of the European Society of Contact Dermatitis. Contact Dermatitis 1990; 22:164-78.

30 Voegeli R, Rawlings a V, Doppler S, et al. Profiling of serine protease activities in human stratum corneum and detection of a stratum corneum tryptase-like enzyme. Int J Cosmet Sci 2007; 29:191-200.

31 Bashir SJ, Chew a L, Anigbogu a, et al. Physical and physiological effects of stratum corneum tape stripping. Skin Res Technol 2001; 7:40-8.

32 Rawlings A V, Canestrari DA, Dobkowski B. Moisturizer technology versus clinical performance. Dermatol Ther 2004; 17 Suppl 1:49-56.

33 Suzuki Y, Nomura J, Hori J, et al. Detection and characterization of endogenous protease associated with desquamation of stratum corneum. Arch Dermatol Res 1993; 285:372-7.

34 Winge MC, Hoppe T, Berne B, et al. Filaggrin genotype determines functional and molecular alterations in skin of patients with atopic dermatitis and ichthyosis vulgaris. PLoS One 2011; 6:e28254.

35 Fluhr J, Bankova LG. Skin Surface pH: Mechanism, Measurement, Importance. In: Handbook of Non-Invasive Methods and the Skin (Serup J, Jemec GB, Grove GL, eds). Boca Raton, CRC Press, 2006; 411-20.

36 Brown K, Higgs-Bayliss T, Chittock J, et al. The effect of an emollient cream containing 5\% urea on the biophysical properties of aged skin. In: Internation Investigative Dermatology. Edinburgh, 2013.

37 Hachem JP, Roelandt T, Schurer N, et al. Acute acidification of stratum corneum membrane domains using polyhydroxyl acids improves lipid processing and inhibits degradation of corneodesmosomes. J Invest Dermatol 2010; 130:500-10. 
38 Kottner J, Lichterfeld A, Blume-Peytavi U. Maintaining skin integrity in the aged: A systematic review. Br J Dermatol 2013; 169:528-42.

39 Danby S, Brown K, Higgs-Bayliss T, et al. The effect of a complex emollient cream containing 5\% urea, ceramide 3 and lactic acid, compared with a simple emollient, on the skin barrier in older people with dry skin. Br J Dermatol 2014; 171:57.

40 Lodén M, Loden M. Effect of moisturizers on epidermal barrier function. Clin Dermatol 2012; 30:286-96.

41 Danby SG. Biological Variation in Skin Barrier Function: From A (Atopic Dermatitis) to X (Xerosis). Curr Probl Dermatol 2016; 49:47-60. 


\section{FIGURES}
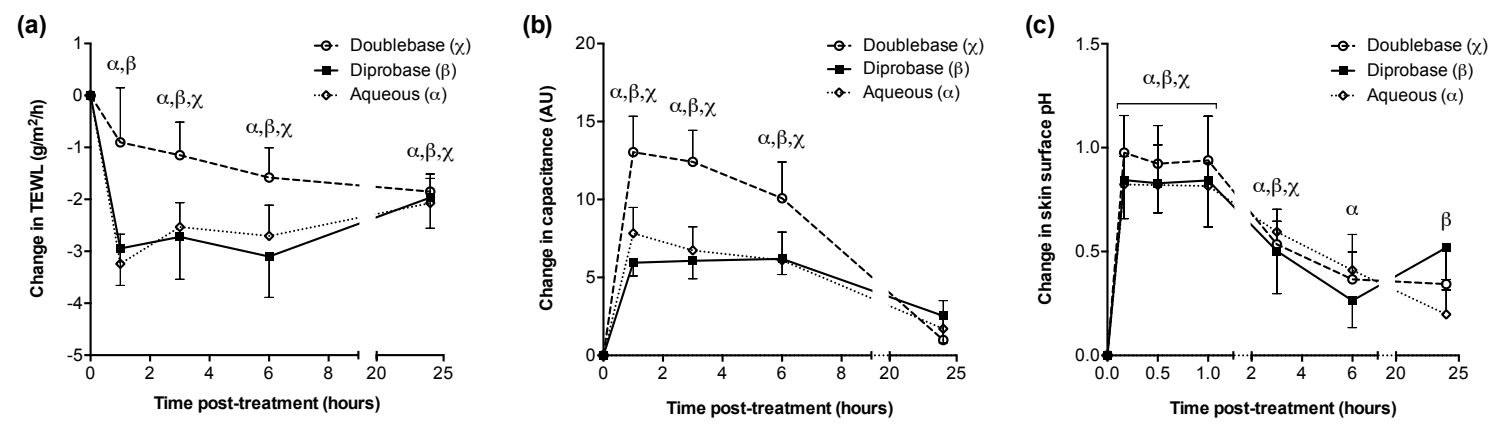

Figure 1: The effect of a single application of Aqueous cream (open circles), Diprobase ${ }^{\circledR}$ cream (closed squares) and Doublebase ${ }^{\mathrm{TM}}$ gel (open diamonds) on skin barrier function (TEWL, panel a), skin hydration (capacitance, panel b), and skin surface $\mathrm{pH}$ (panel c). Results are presented as the change compared to untreated skin. There are 8 repeats of each treatment in 4 subjects. For all three measured parameters a significant effect of the treatment and the interaction between the treatment and time-post washing was found ( $p<0.05,2$-way repeated measures ANOVA). Symbols $(\alpha, \beta, \gamma)$ indicate the results of a Dunnett post-test comparing all treatments to the untreated control. 
(a)

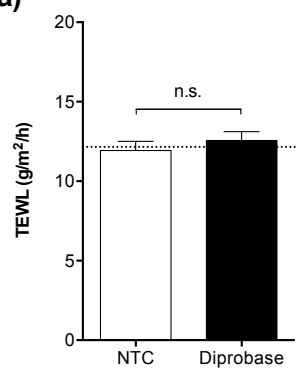

(b)

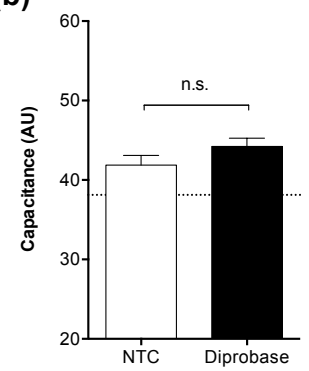

(c)

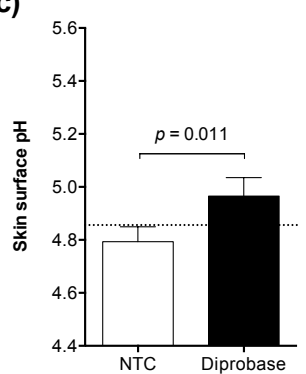

(d)

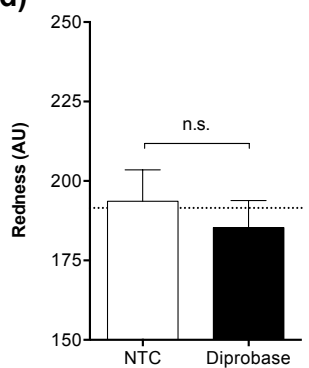

(e)

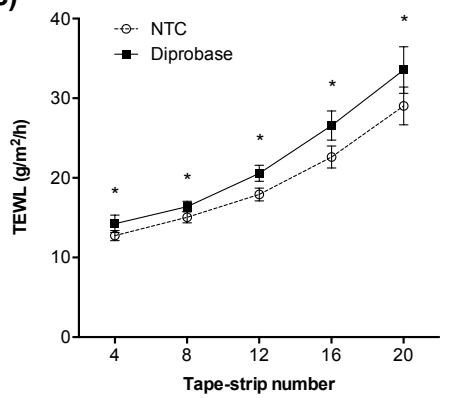

(f)

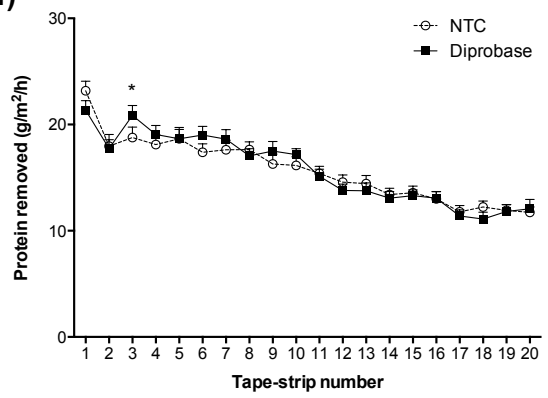

(g)

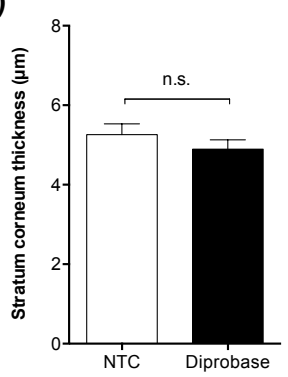

(h)

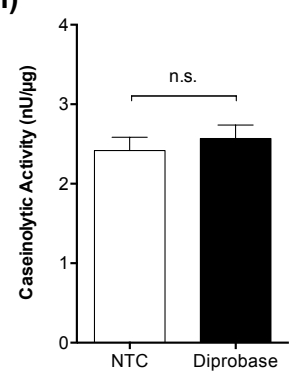

(i)

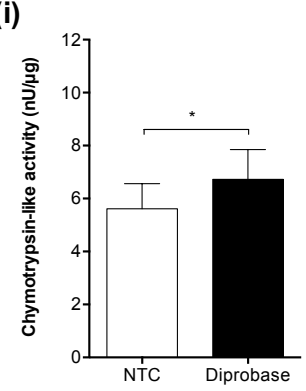

(j)

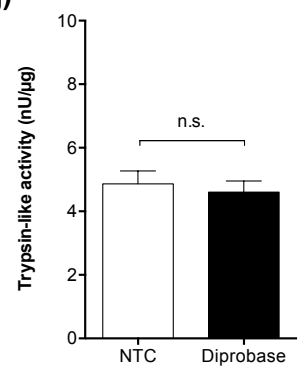

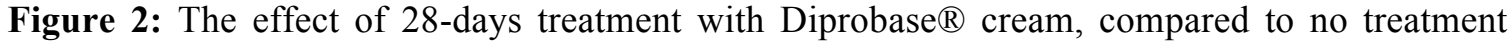
(NTC), on the biophysical and biological properties of the skin in people with quiescent AD. (a) Skin barrier function, (b) skin hydration, (c) skin surface pH, (d) objective erythema, (e) SC integrity measured as TEWL in conjunction with tape-stripping, (f) SC cohesion (protein removed by tape-stripping), (g) estimated SC thickness, (h) ex vivo SC broad-spectrum caseinolytic protease activity, (g) chymotrypsin-like protease activity, (h) and trypsin-like activity. TEWL, capacitance, skin surface $\mathrm{pH}$ and redness were compared with ANCOVA using baseline (Day 0) values as the covariant. SC integrity and cohesion were assessed using a repeated measures 2-way ANOVA. Both the treatment $(p<0.0001)$ and the number of tape-strips significantly $(p=0.0002)$ affected SC integrity, but not the interaction between the two (n.s.). The number of tape-strips $(p<0.0001)$ and the interaction between tape-strip number and treatment $(p=0.0009)$ affected SC cohesion, but not the treatment independently (n.s.). SC thickness and caseinolytic, chymotrypsin-like and trypsin-like activities were compared using a paired t-test. *Significant differences using either a t-test (a-d and g-j) or Bonferroni post-test (e and f). n.s., not significant. 
(a)

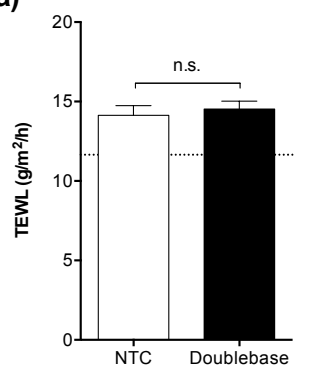

(b)

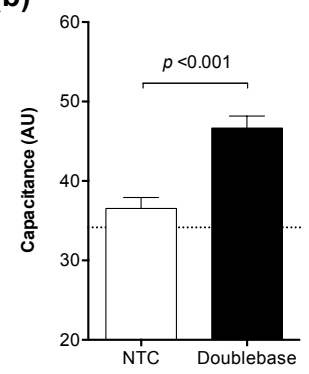

(c)

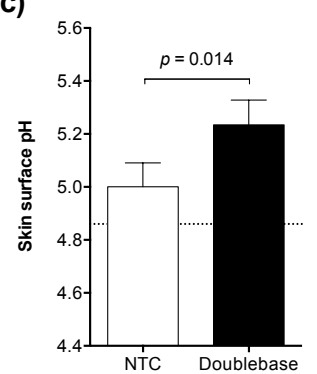

(d)

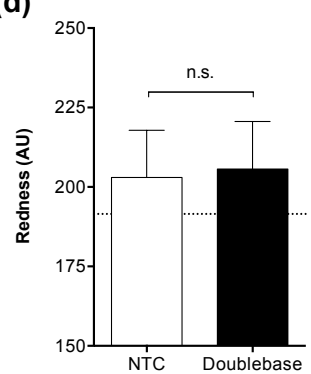

(g)

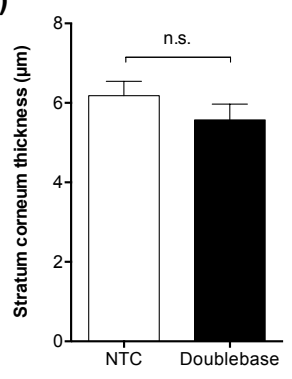

(e)

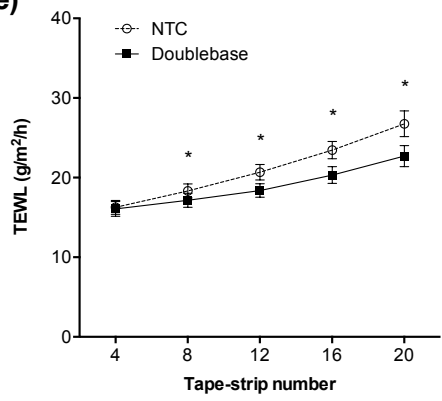

(f)

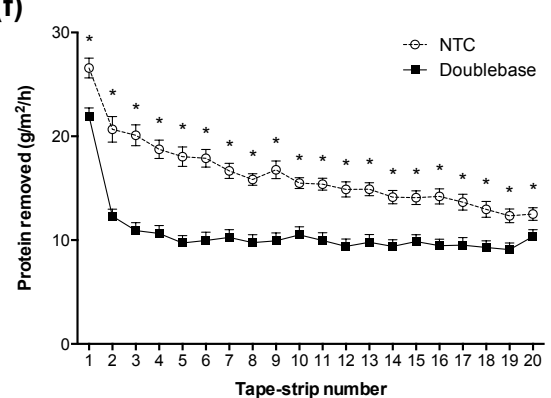

(j)

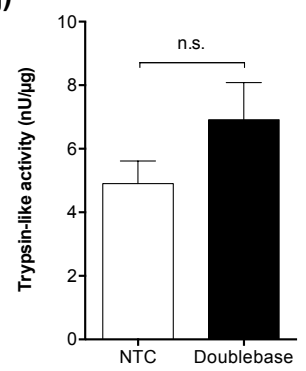

(h)

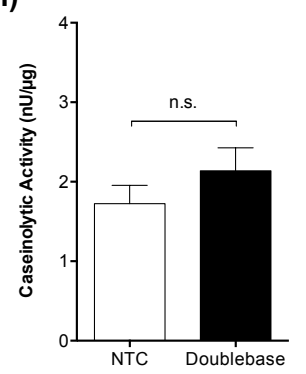

(i)

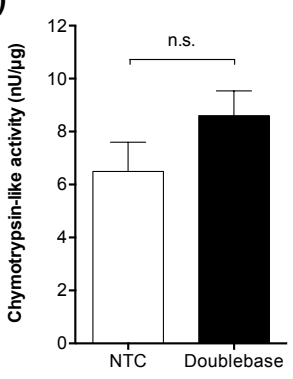

pe-strip number

Figure 3: The effect of 28-days treatment with Doublebase ${ }^{\mathrm{TM}}$ gel, compared to no treatment (NTC), on the biophysical and biological properties of the skin in people with quiescent AD. (a) Skin barrier function, (b) skin hydration, (c) skin surface pH, (d) objective erythema, (e) SC integrity measured as TEWL in conjunction with tape-stripping, (f) SC cohesion (protein removed by tape-stripping), (g) estimated SC thickness, (h) ex vivo SC broad-spectrum caseinolytic protease activity, (g) chymotrypsin-like protease activity, (h) and trypsin-like activity. TEWL, capacitance, skin surface $\mathrm{pH}$ and redness were compared with ANCOVA using baseline (Day 0) values as the covariant. SC integrity and cohesion were assessed using a repeated measures 2-way ANOVA. The treatment $(p=0.0003)$, the number of tape-strips $(p<0.0001)$ and the interaction between the 2 factors significantly $(p<0.0001)$ affected SC integrity. The treatment $(p<0.0001)$, the number of tape-strips $(p<0.0001)$ and the interaction between the 2 factors significantly $(p<0.0001)$ affected SC cohesion. SC thickness and caseinolytic, chymotrypsin-like and trypsin-like activities were compared using a paired t-test. *Significant differences using either a t-test (a-d and g-j) or Bonferroni post-test (e and f). n.s., not significant. 